\title{
Regulation of Contractile Proteins and Protein Translational Signaling in Disused Muscle
}

\author{
Hua Liu, a,dh Eric R. Blough ${ }^{a, b, h} \quad$ Ravikumar Arvapallia Yeling Wang ${ }^{a, e}$ \\ Peter J. Reiser ${ }^{f}$ Satyanarayana Paturia Anjaiah Katta ${ }^{a}$ Robert Harris ${ }^{9}$ \\ Niraj Nepalg Miaozong Wu ${ }^{a, b, c}$
}

${ }^{a}$ Center for Diagnostic Nanosystems; ${ }^{b}$ School of Pharmacy; ' Department of Internal Medicine, Joan C. Edwards School of Medicine, Marshall University, Huntington, WV; dDepartment of Physiology and Pharmacology, Southeast University, Nanjing; eThe First Hospital, Jilin University, Jilin; fDepartment of Oral Biology, Ohio State University, Columbus, $\mathrm{OH}_{\text {; }}{ }^{\text {Department }}$ of Biology, West Virginia State University, Institute, WV; hLiu H and Blough ER have contributed equally to this work

\section{Key Words}

Skeletal muscle atrophy $•$ Unloading $•$ Contractile proteins $•$ Protein translational signaling

\begin{abstract}
Background/Aims: Muscle disuse can lead to muscle atrophy and impaired skeletal muscle function. How skeletal muscle modulates protein translational signaling in response to prolonged muscle disuse is not well understood. Using the hindlimb unloading (HU) model of muscle atrophy we examined how hindlimb unweighting affects protein translational signaling, including the activation of Akt/mTOR/p70S6K/S6 signaling and the inhibitory association of 4EBP1 with translation initiation factor eIF4E. Methods: Male F344BN rats were randomized into baseline control, or subjected to $\mathrm{HU}$ for 3, 7 or 14 days. Body weight, gastrocnemius muscle, and individual myofiber cross-sectional area were measured to evaluate the degree of muscle atrophy. The amounts of myosin and related muscle contractile proteins were assessed using SDS-PAGE and immunoblotting. Microarray analysis was used to evaluate changes in the mRNA expression of muscle contractile proteins. Total and phosphorylated proteins of $\mathrm{Akt} / \mathrm{mTOR} / \mathrm{p} 70 \mathrm{~S} 6 \mathrm{~K} / \mathrm{S} 6$ pathway were determined via immunoblotting, while the association of 4EBP1 with eIF4E was detected via co-immunoprecipitation. Results: Unloading for 3 days significantly reduced cytosolic myosin content and was associated with increased binding of 4EBP1 to eIF4E, while prolonged unloading (14 days) was associated with the activation of Akt/mTOR/p70S6K/S6 signaling, decreased binding of 4EBP1 to eIF4E, increased cytosolic myosin and elevations in myofibrillar mRNA levels. Conclusion: Taken together, these data suggest that prolonged muscle disuse induces a biphasic translational signaling response that is associated with diminished and then increased muscle contractile protein expression.
\end{abstract}




\section{Introduction}

Muscle disuse such as that encountered during extended bed rest, muscle immobilization or spaceflight can lead to muscle atrophy and impaired muscle function [1-3]. The loss or accumulation of muscle contractile protein is determined by the balance between muscle protein degradation and synthesis. The Akt / mTOR (the protein kinase B / mammalian target of rapamycin) pathway is an important regulator of protein synthesis and plays a critical role in the stimulation of myocyte growth $[4,5]$. The regulation of protein translation by Akt/mTOR signaling is effected through the phosphorylation (activation) of p70S6 kinase (p70S6K) which functions to phosphorylate / activate the S6 ribosomal protein, and the phosphorylation (inactivation) of the eukaryotic translation initiation factor eIF4E binding protein-1 (4EBP1) [6-8]. Once phosphorylated, 4EBP1 undergoes dissociation from eIF4E which allows for the initiation of mRNA cap-dependent translation [9-11]. The phosphorylation and interaction of molecules thought to participate in the regulation of muscle atrophy produced by hindlimb unloading are not well understood.

The rat and mouse hindlimb unloading (HU) models have been widely used to study the muscle atrophy associated with disuse and weightlessness [1-3]. Herein we examine how $\mathrm{HU}$ for fourteen days affects the expression of muscle contractile proteins and the regulation of protein translational signaling. We hypothesized that HU would initially cause a down regulation of protein translational signaling but that prolonged HU would be associated with activated protein translational signaling as the muscle began to remodel the sarcomere. We found that muscle contractile protein levels and the activation of translational signaling were diminished during the acute phase of unloading (0-3 days) but that 14 days of HU stimulated an increase in the abundance of muscle contractile proteins in myofibrillar soluble fraction, with this latter event appearing to be regulated, at least partially, by the activation of Akt/ mTOR/p70S6K/S6 pathway and decreased 4EBP1-eIF4E interaction. These data provide new information regarding the molecular signaling of muscle remodeling during decreases in contractile loading.

\section{Materials and Methods}

\section{Materials}

Primary antibodies of myosin heavy chain, M8421 and MF-20, were from Sigma-Aldrich (St. Louis, MO) and Developmental Studies Hybridoma Bank (Iowa City, IA), respectively. Dystrophin primary antibody (NCL-DYS2) was purchased from Novocastra Vector Laboratories (Burlingame, CA). Antibodies for Akt (\#9272), phospho-Akt (Thr308) (pAkt-Thr308, \#9275), phospho-Akt (Ser473) (pAkt-Ser473, \#9271), mTOR (\#2972), phospho-mTOR (Ser2448) (p-mTOR, \#2971), p70S6 Kinase (p70S6K; \#9202), phosphop70S6 Kinase (Thr389) (p-p70S6K; \#9205), S6 ribosomal protein (\#2217), phospho-S6 ribosomal protein (Ser235/236) (pS6, \#2211), 4EBP1 (\#9644 and \#9452), phospho-4EBP1 (Thr37/46) (\#9459), eIF4E (\#2067), GAPDH (glyceraldehyde 3-phosphate dehydrogenase, \#2118), secondary antibodies (anti-rabbit (\#7074) or anti-mouse (\#7076)) that was conjugated with horseradish peroxidase (HRP) were from Cell Signaling Technology (Beverly, MA). Pierce tissue protein extraction reagent (T-PER), Pierce protein assay reagent (\#22660), GE Healthcare Amersham ECL ${ }^{\mathrm{TM}}$ Western Blotting Detection kits (RPN 2106 and RPN2135) were from Thermo Fisher Scientific Inc. (Rockford, IL). Protein A/G PLUS Agarose (sc-2003) were purchased from Santa Cruz Biotechnology (Santa Cruz, CA). The PAGEr Gold Precast gels (10\% and $15 \%$ ) were from Lonza (Rockland, ME). RAPIDstain ${ }^{\text {TM }}$ for protein staining based on Coomassie dye was from G-Biosciences (St. Louis, MO). VECTASHIELD HardSet Mounting Medium with DAPI was purchased from Vector Laboratories (Burlingame, CA). Dual Color protein molecular weight marker was from BioRad (Hercules, CA). Protease inhibitors (\#P8340), phosphatase inhibitors (\#P5726), Laemmli $4 \times$ sample buffer, poly-lysine and all other chemicals were purchased from Sigma-Aldrich (St. Louis, MO). Tincture of Benzoin aerosol spray was from Professional Packaging Corp (Aurora, IL). Skin-Trac adhesive traction strips were from Zimmer, Inc. (Warsaw, IN). RNeasy Mini Kit for RNA isolation and RNeasy MinElute Cleanup Kit were from Qiagen (Valencia, CA). Ambion Amino Allyl MessageAmp ${ }^{\text {TM }}$ II Kit was from Life Technologies (Carlsbad, 
CA). Amersham Cy3 and Cy5 Maleimide mono-Reactive Dye was from GE Healthcare (Piscataway, NJ). CodeLink $^{\text {TM }}$ Rat Whole Genome Bioarray was from Applied Microarrays (Tempe, AZ).

\section{Animals, hindlimb unloading procedure and tissue collection}

The experimental procedures and animal care were approved and conducted in accordance with the Institutional Animal Care and Use Committee at Marshall University. Fischer 344/NNiaHSd x Brown Norway/ BiNia F1 hybrid rats (F344BN; Male, aged 3 months and weighed $324.7 \pm 6.3 \mathrm{gm}$ ) were obtained from the National Institute on Aging (Bethesda, MD). Rats were housed in an AAALAC (Association for Assessment and Accreditation of Laboratory Animal Care International)- approved vivarium with $12 \mathrm{~h}-12 \mathrm{~h}$ light-dark cycle and maintained at $22 \pm 2^{\circ} \mathrm{C}$. Food and water were given to the rats ad libitum. Before experimentation, animals were allowed to recover from shipping stress and acclimate to the housing facilities for 2 weeks.

Animals were randomly assigned into four groups of four animals each ( $\mathrm{N}=4)$ : baseline control, hindlimb unloading for 3, 7 and 14 days (HU3, HU7 and HU14, respectively). Muscle atrophy was induced via a hindlimb unloading procedure developed by the NASA Ames Research Center [3]. Briefly, animal tail was cleaned with alcohol, and then allowed to dry before applying the Tincture of Benzoin. After the Benzoin was dry, Skin-Trac adhesive traction strips were applied on two sides of the tail up to 2/3 tail length. The body of animal was suspended about a $30^{\circ}$ angle from the cage floor to assure that the both hindlimbs did not touch the grid floor. The suspended animal was free to move and rotate $360^{\circ}$ using forelimbs, and had free access to food and water. Total food consumption was measured and the daily food intake was calculated.

After completion of unloading procedure, animals were anesthetized using a ketamine-xylazine (4:1) cocktail (50 mg/kg, i.p). Body weight was recorded. Gastrocnemius muscle was removed from anesthetized rats, weighed, frozen in liquid nitrogen, and stored at $-80^{\circ} \mathrm{C}$. The middle cross-section of gastrocnemius muscle ( $\sim 2 \mathrm{~mm}$ thickness) was used for tissue section, while the rest of gastrocnemius muscle was pulverized using a Bessman tissue pulverizer (Spectrum Laboratories; Rancho Dominguez, CA), mixed well and aliquoted for protein and RNA extractions.

\section{Determination of myofiber cross-sectional area (CSA)}

Gastrocnemius muscle was serially sectioned $(8 \mu \mathrm{m})$ using an IEC Minotome cryostat, and collected on glass slides coated with poly-lysine. Immunohistochemistry with anti-dystrophin antibody was performed as described previously $[12,13]$. Myofiber CSA was determined by tracing the outline of dystrophin-stained fibers using the ImageJ program (http://rsbweb.nih.gov/ij/) as outlined elsewhere [13]. The distribution of myofiber CSA was plotted with Vertical Box and Whisker Plots, and multiple comparisons were used to determine differences of means between groups.

\section{Microarray analysis}

Total RNA was isolated from gastrocnemius muscles of HU3 and HU14 rats using the Qiagen RNeasy Mini Kit. RNA concentration was determined using a Nanodrop spectrophotometer and the integrity was assessed using an Agilent 2100 Bioanalyzer. The amplified RNA (aRNA) preparation was performed using the Amino Allyl MessageAmp ${ }^{\mathrm{TM}}$ II kit as detailed by the manufacturer. The aRNA was then coupled to either a Cy3 or Cy5 Maleimide mono-Reactive dye. After purifying with an RNeasy MinElute Cleanup Kit and determining the concentration, $\mathrm{Cy} 3$ and $\mathrm{Cy} 5$ labeled targets were used for microarray hybridization using a CodeLink ${ }^{\mathrm{TM}}$ Rat Whole Genome Bioarray, which was printed with $\sim 34,000$ transcripts and EST's including $\sim 29,000$ well substantiated rat genes. The array was repeated with three independent replicate slides. Image acquisition was performed using a Gene Pix 4100 DNA Microarray Scanner (Agilent Technologies) at $635 \mathrm{~nm}$ and $532 \mathrm{~nm}$ wavelength to detect emission from Сy3 and Cy5 photoreactive dyes, respectively. Up- and down-regulated transcripts in each array were determined using the Ratio of Medians with a lower cut-off threshold of 2.0 and an upper cut-off threshold of 0.5 , respectively.

\section{Extraction of total myofibrillar proteins and analysis by SDS-PAGE}

The sodium dodecyl sulfate-polyacrylamide gel electrophoresis (SDS-PAGE) coupling with silver staining was used to determine the abundance of myosin-related proteins in gastrocnemius muscle. Whole myofibrillar protein fractions were prepared using a sample buffer containing $8 \mathrm{M}$ urea and $2 \mathrm{M}$ thiourea in order to dissolve and extract all myofibrillar proteins as described previously [14]. Proteins were 
Liu/Blough/Arvapalli et al.: Muscle Disuse Changes Contractile Proteins and Translational Signaling

separated using 12\% polyacrylamide gels in Hoefer SE600 gel units and run at constant current (25 mA/ gel) as described [15]. The gels were fixed using 50\% methanol / 10\% acetic acid and then silver-stained using a solution containing $46 \mathrm{mM}$ silver nitrate as outlined previously [14,15]. Gels were scanned using a Hoefer model GS 300 densitometer equipped with GS365W software (ver. 3.01). Myosin heavy chain (MHC), myosin light chain-1 fast and - 2 fast (MLC-1F and $2 \mathrm{~F}$ ), and troponin isoform C, I and T (TnnC, TnnI and TnnT) were identified based on molecular size and band migration pattern [15].

\section{Extraction of cytosolic proteins and analysis by SDS-PAGE}

The cytosolic (soluble) proteins were extracted from a proportion of pulverized gastrocnemius muscle using a mild buffer, T-PER, which contained phosphatase inhibitors (P5726; 1:100) and protease inhibitors (P8340; 1:100) as described previously $[12,16]$. Protein concentration was determined using a Pierce $660 \mathrm{~nm}$ protein assay reagent (\#22660). The proteins were boiled in the Laemmli $4 \times$ sample buffer for 5 min. Proteins were separated using $10 \%$ or $15 \%$ PAGEr Gold Precast gels, and the gel was stained with RAPIDstain $^{\text {TM }}$ for protein staining based on Coomassie dye, and scanned for further densitometry analysis.

\section{Immunoblotting analysis}

Immunoblotting analysis was performed to detect target proteins as detailed previously [13,17]. Briefly, T-PER prepared protein samples were separated on the PAGEr Gold Precast gels, and then transferred to nitrocellulose membranes. After incubating with primary antibody at $4{ }^{\circ} \mathrm{C}$ overnight and secondary antibody for $1 \mathrm{~h}$ at room temperature, target proteins were visualized following reaction with $\mathrm{ECL}^{\mathrm{TM}}$ Reagents. The abundance of target protein was quantitated using AlphaEaseFC image analysis software (Alpha Innotech, San Leandro, CA) and then normalized to GAPDH. The relative abundance of phosphorylated protein was normalized to its total protein and the GAPDH.

\section{Determination of eIF4E / 4EBP1 association}

The interaction of 4EBP1 with eIF4E was determined by co-immunoprecipitation as described elsewhere [16]. Briefly, $200 \mathrm{mg}$ of T-PER prepared protein homogenate (180 $\mu \mathrm{L})$ was incubated with eIF4E primary antibody (1:50 dilution) and protein A/G PLUS Agarose $(20 \mu \mathrm{L})$ overnight at $4{ }^{\circ} \mathrm{C}$. Agarose beads were then collected via an $8,000 \mathrm{~g}$ centrifugation for $30 \mathrm{sec}$ at $4{ }^{\circ} \mathrm{C}$, and washed with $500 \mu \mathrm{L}$ of ice-cold T-PER buffer with protease and phosphatase inhibitors for three times. The protein bound with beads was released by boiling in $40 \mu \mathrm{L}$ of Laemmli $2 \times$ sample buffer for $5 \mathrm{~min}$. Twenty microliters of each supernatant were subjected to immunoblotting analysis for 4EBP1 detection.

\section{Data analysis}

Results are presented as mean \pm standard error (SE). The effects of unloading were analyzed using the SAS GLM procedure (SAS Institute Inc., Cary, NC). Multiple comparisons using the Tukey-Kramer test were performed to determine differences between groups. The level of significance accepted $a$ priori was $\leq 0.05$.

\section{Results}

Muscle mass and myofiber cross-sectional area

Hindlimb unloading resulted in loss of body weight $(\mathrm{P} \leq 0.05$; Fig. $1 \mathrm{~A})$. The ratio of gastrocnemius muscle / body weight was not statistically different between baseline control and HU rats for 3 days, while prolonged unloading for 7 and 14 days progressively decreased the muscle / BW ratio ( $\mathrm{P} \leq 0.05$; Fig. 1B). Myofiber cross-sectional area (CSA) was not different between the control and 3-day unloaded animals, while it became progressively decreased in the 7- and 14-day unloaded animals ( $\mathrm{P} \leq 0.05$; Fig. $1 \mathrm{C}$ ). Compared to the baseline control animals, daily food intake in the unloaded animals was less( $\mathrm{P} \leq 0.05$; Fig. 1D).

\section{Myofibrillar transcript expression}

Compared to that observed in the 3-day unloaded animals, microarray analysis demonstrated that the mRNA expression of 272 genes was increased, while expression of 812 genes was decreased in the HU14 muscle. The abundance of mRNA transcripts for myosin 


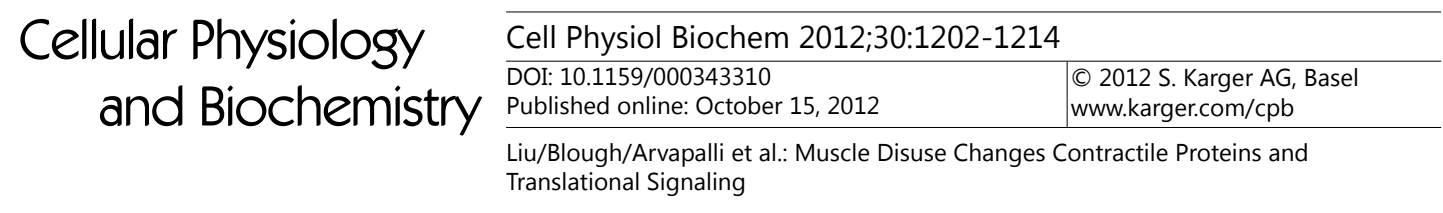

Fig. 1. Effect of hindlimb unloading on gastrocnemius muscle mass and myofiber crosssectional area (CSA). A. Body weight loss after 3,7 and 14 days of hindlimb unloading (HU). B. The percentage of gastrocnemius muscle to body weight (BW). C. Myofiber cross-sectional area (CSA, $\mu \mathrm{m}^{2} /$ fiber). Left panel: Representative images of dystrophinstained gastrocnemius muscle section from baseline and rats unloaded for 3,7 or 14 days. Right panel: Vertical box and
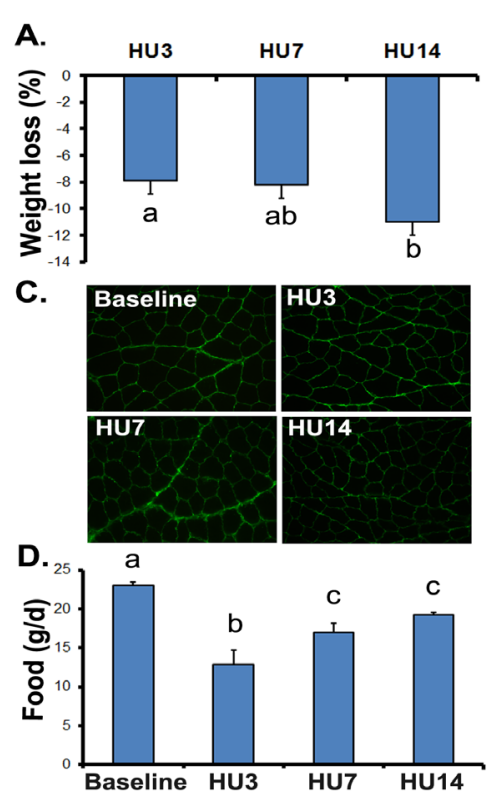

B.
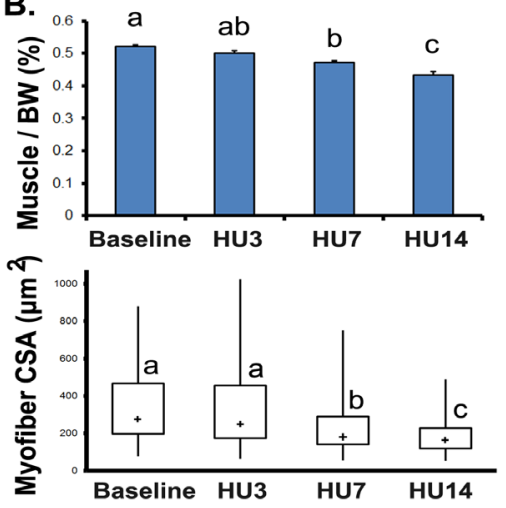

whisker plots to show the distribution of myofiber CSA: median values ( + ), the 25 th and 75 th percentile (the bottom and top of box, respectively), and the minimum and maximum (the bottom and top end of whisker, respectively). Number of myofibers measured for baseline and rats unloaded for 3, 7 and 14 days was 1125, 1265, 1344 and 1349, respectively, from 4 animals in each group. D. Daily food consumption. abc: Groups without the same letter are significantly different $(\mathrm{P} \leq 0.05)$.

Table 1. Expression profile of myofibrillar related genes: microarray data

\begin{tabular}{|c|c|c|c|}
\hline Symbol & Name & $\begin{array}{c}\text { Fold } \\
\text { (HU14/HU3) }\end{array}$ & Protein function \\
\hline MLC1 & myosin, light polypeptide 1 & 2.7 & Structural constituent of muscle \\
\hline MLCpf & $\begin{array}{l}\text { myosin light chain (phosphorylatable), } \\
\text { fast skeletal muscle }\end{array}$ & 2.4 & Structural constituent of muscle \\
\hline Mybpc1 & myosin binding protein $C$, slow type & 2.3 & Structural component of skeletal muscle \\
\hline Mybph & myosin binding protein $\mathrm{H}$ & 2.6 & skeletal muscle structural constituent \\
\hline Acta1 & actin, alpha 1 , skeletal muscle & 3.2 & Structural component of muscle contractile apparatus \\
\hline Ttn & titin & 2.8 & Skeletal muscle structural constituent \\
\hline Nexn & nexilin ( $\mathrm{F}$ actin binding protein) & 2.4 & Actin filament binding protein \\
\hline Xirp1 & xin actin-binding repeat containing 1 & 2.6 & Actin binding protein \\
\hline Nrap & nebulin-related anchoring protein & 2.6 & Muscle alpha-actinin binding \\
\hline TnnI2 & troponin I type 2 (skeletal, fast) & 3.1 & $\begin{array}{l}\text { Bind to actin in thin myofilaments to regualte troponin-tropomyosin } \\
\text { complex }\end{array}$ \\
\hline TnnC2 & troponin C type 2 (fast) & 4.3 & Calcium binding to produce a conformational change in TnnI \\
\hline
\end{tabular}

and actin and several other myofibrillar proteins was increased over 2-fold after 14 days of unloading compared to that observed in animals that had been subjected to unloading for 3 days (Table 1). 


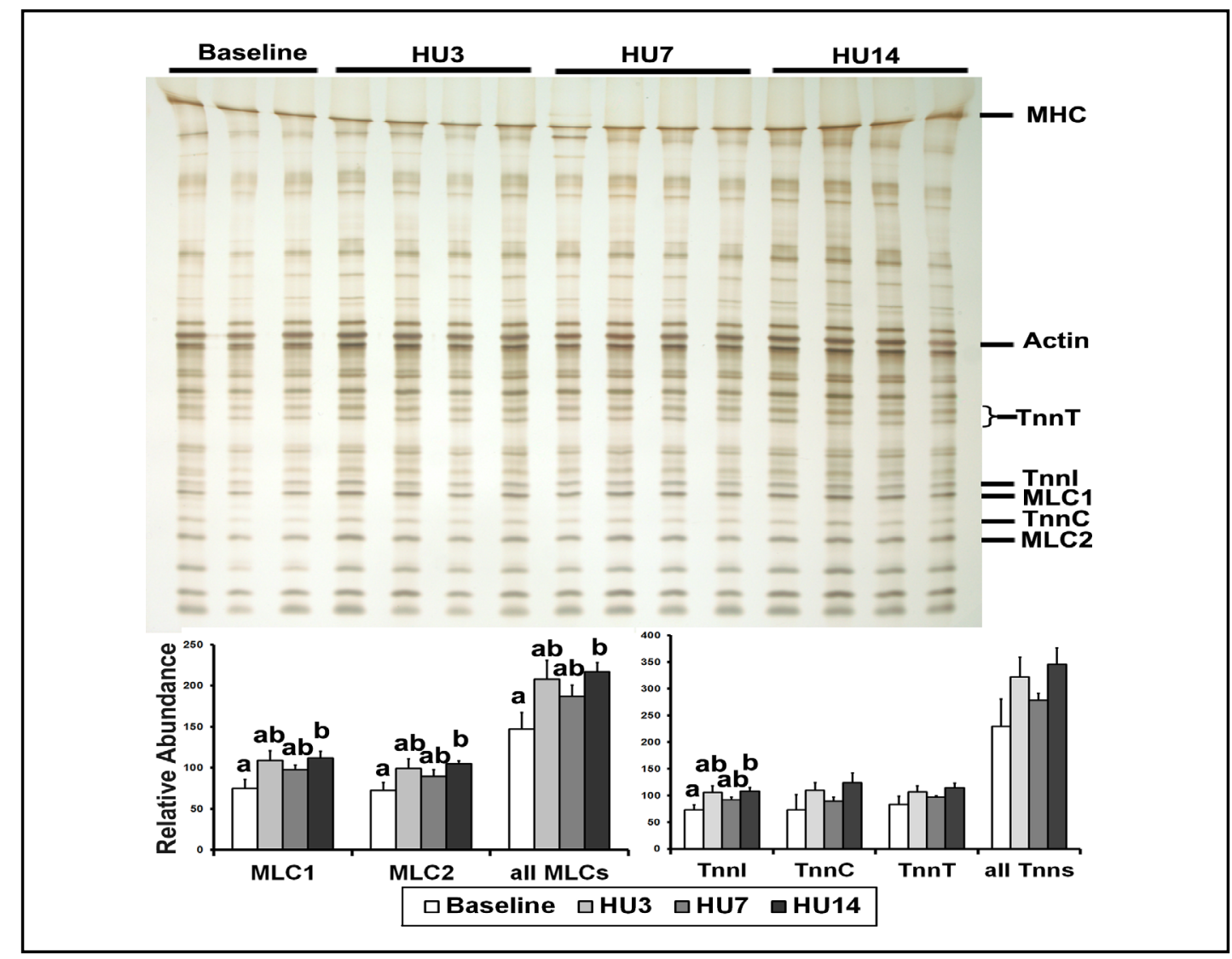

Fig. 2. Effect of hindlimb unloading on muscle contractile proteins in gastrocnemius muscle. Total muscle protein was prepared, as described in Methods, and subjected to SDS-PAGE, followed by silver staining. Due to size limitations associated with a 15-well comb only 3 baseline protein samples were loaded. MHC: myosin heavy chain; MLC1 and MLC2: myosin light chain-1 fast and -2 fast; TnnC, TnnI and TnnT: troponin $\mathrm{C}$ fast, I fast and $\mathrm{T}$ fast. ab: Groups without the same letter are significantly different $(\mathrm{P} \leq 0.05)$.

Myosin-related proteins in whole myofibrillar proteins

In an effort to extend our microarray data, we next examined how unloading affected the expression of myofibrillar proteins. To this end, we used a protein extraction buffer containing $8 \mathrm{M}$ urea and $2 \mathrm{M}$ thiourea that was specifically formulated to extract all myofibrillar proteins [14]. Myosin heavy chain, actin, myosin light chain and troponin isoforms were separated and identified using a 12\% SDS-PAGE gel. Compared to control animals, myosin light chains (including MLC1 and MLC2) and troponin isoform I (TnnI) were more abundant after 14 days of unloading (Fig. 2).

\section{Myosin in myofibrillar cytosolic fraction}

It has been suggested that the myofibrillar soluble protein fraction is altered with muscle growth or atrophy [18]. To examine this possibility, soluble proteins were isolated using a mild T-PER extraction buffer and subjected to SDS-PAGE. Coomassie staining suggested that the protein abundance at $\sim 200 \mathrm{kDa}$ was significantly decreased in the 3-day HU animals, while it was increased in the 14 -day HU samples ( $\mathrm{P} \leq 0.05$; Fig. $3 \mathrm{~A}$ ). As the molecular weight of myosin heavy chain is around $\sim 200 \mathrm{kDa}$, we further used a myosin-specific antibody (M8421 from Sigma-Aldrich) to detect the difference of myosin in the myofibrillar soluble protein fractions. As shown in Figure 3B, the abundance of myosin heavy chain was decreased in the 3-day HU muscles, while it was increased in the soluble protein fractions from the 


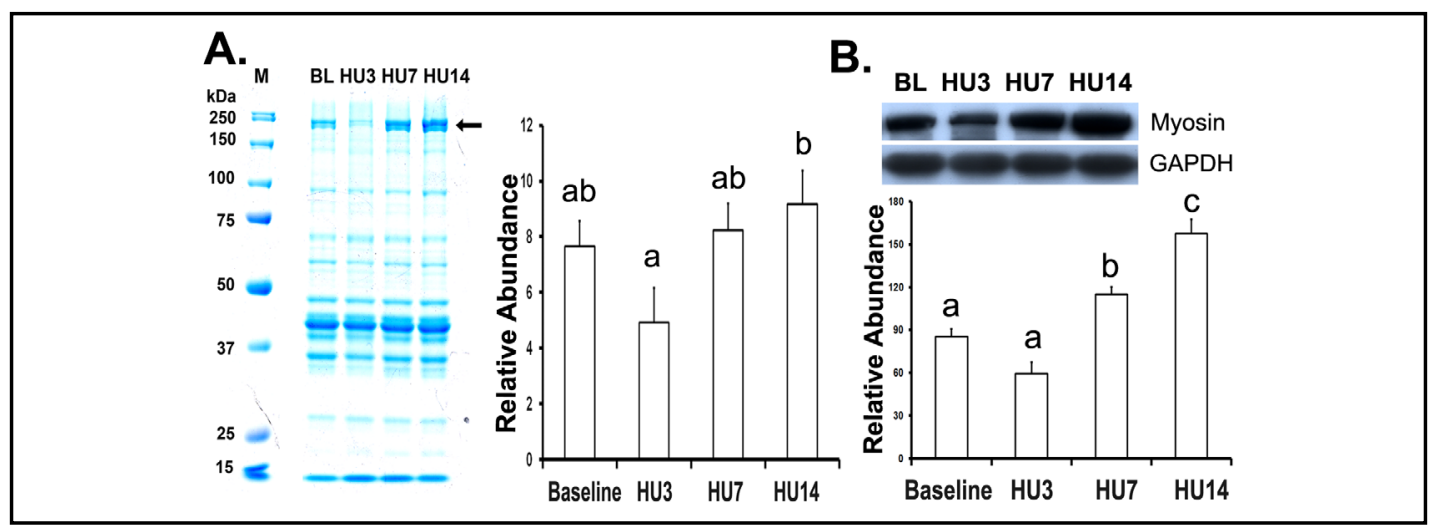

Fig. 3. Effect of hindlimb unloading on myosin in the cytosolic protein from gastrocnemius muscle. A. Coomassie staining. Proportion of gastrocnemius muscle tissue was subjected to a cytosolic soluble protein extraction protocol using the T-PER buffer. Myofibrillar proteins were separated using SDS-PAGE then followed by the Coomassie staining. Left panel: Representative images of SDS-PAGE. M: protein marker; BL: baseline; Arrow indicates a significant change of protein band at $\sim 200 \mathrm{kDa}$. Right panel: Relative abundance of $\sim 200 \mathrm{kDa}$ protein bands to all protein bands of the same sample. B. Relative abundance of myosin heavy chain protein in myofibrillar cytosolic protein sample detected by immunoblotting. abc: Groups without the same letter are significantly different $(P \leq 0.05)$.

Fig. 4. Effect of hindlimb unloading on the phosphorylation of Akt and mTOR. A. Phosphorylation of Akt at Ser473 and Thr308 in cytosolic proteins of gastrocnemius muscle determined by immunoblotting. There was no difference in total Akt expression between groups $(\mathrm{P}=0.47)$. B. Phosphorylation of mTOR at Ser2448. ab: Groups without the same letter are significantly different $(\mathrm{P} \leq 0.05)$.

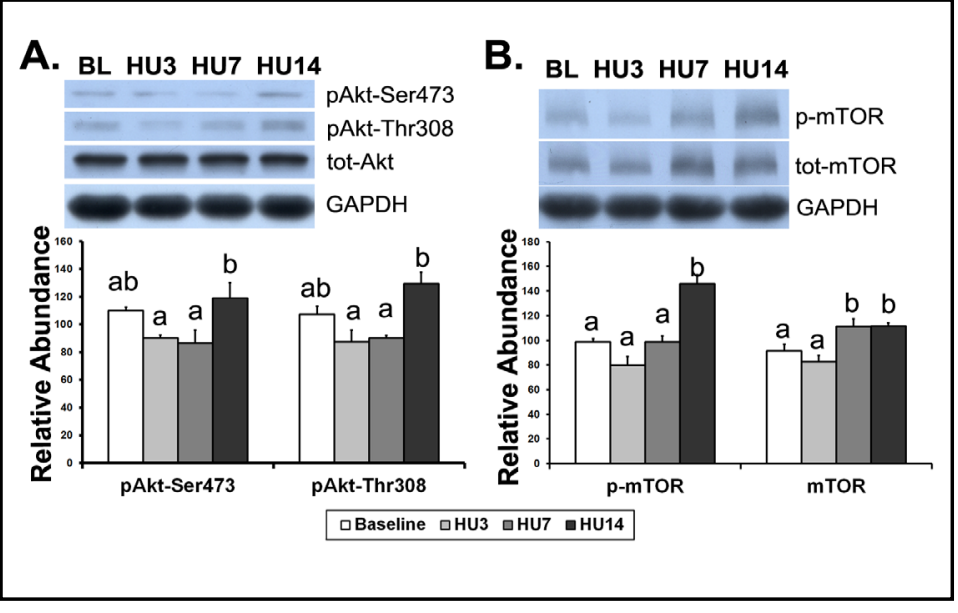

7- and 14-day HU rats. This result was further verified using another anti-myosin antibody (MF-20; Developmental Studies Hybridoma Bank), and the identical result was found (data not shown).

\section{Phosphorylation of Akt/mTOR signaling}

Compared to the control animals, the phosphorylation of Akt at both Ser473 and Thr308 was not changed in the gastrocnemius cytosolic soluble protein fractions obtained from the 3 and 7 days of HU animals, while it was increased in the animals that underwent HU for 14 days ( $\mathrm{P} \leq 0.05$; Fig. 4A). The phosphorylation of mTOR at Ser 2448 was not changed in the 3 - and 7-day HU muscles, while it was increased in the 14-day HU muscles ( $\mathrm{P} \leq 0.05$; Fig. 4B).

\section{Phosphorylation of $p 7056 \mathrm{~K} / \mathrm{S6}$ signaling}

The phosphorylation of p70S6K at Thr389 was not changed in the gastrocnemius cytosolic soluble protein fractions from 3- and 7-day HU rats, while it was increased 
Fig. 5. Effect of hindlimb unloading on phosphorylation of $\mathrm{p} 70 \mathrm{~S} 6 \mathrm{~K}$ and $\mathrm{S6}$. A. Phosphorylation of $\mathrm{p} 70 \mathrm{~S} 6 \mathrm{~K}$ at Thr389 in cytosolic proteins of gastrocnemius muscle as determined by immunoblotting. B. Phosphorylation of S6 ribosomal protein at Ser235/236. ab: Groups without the same letter are significantly different $(\mathrm{P} \leq 0.05)$.

A. BL HU3 HU7 HU14

\section{B. BL HU3 HU7 HU14}
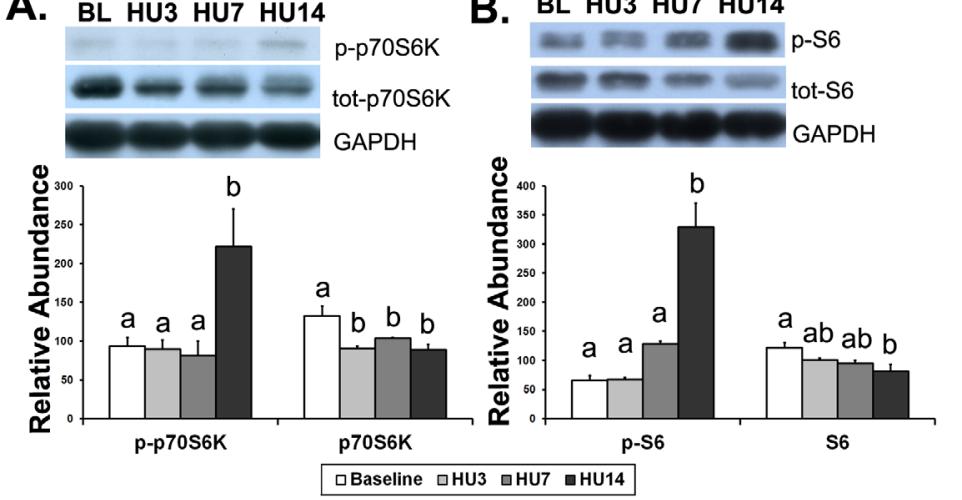

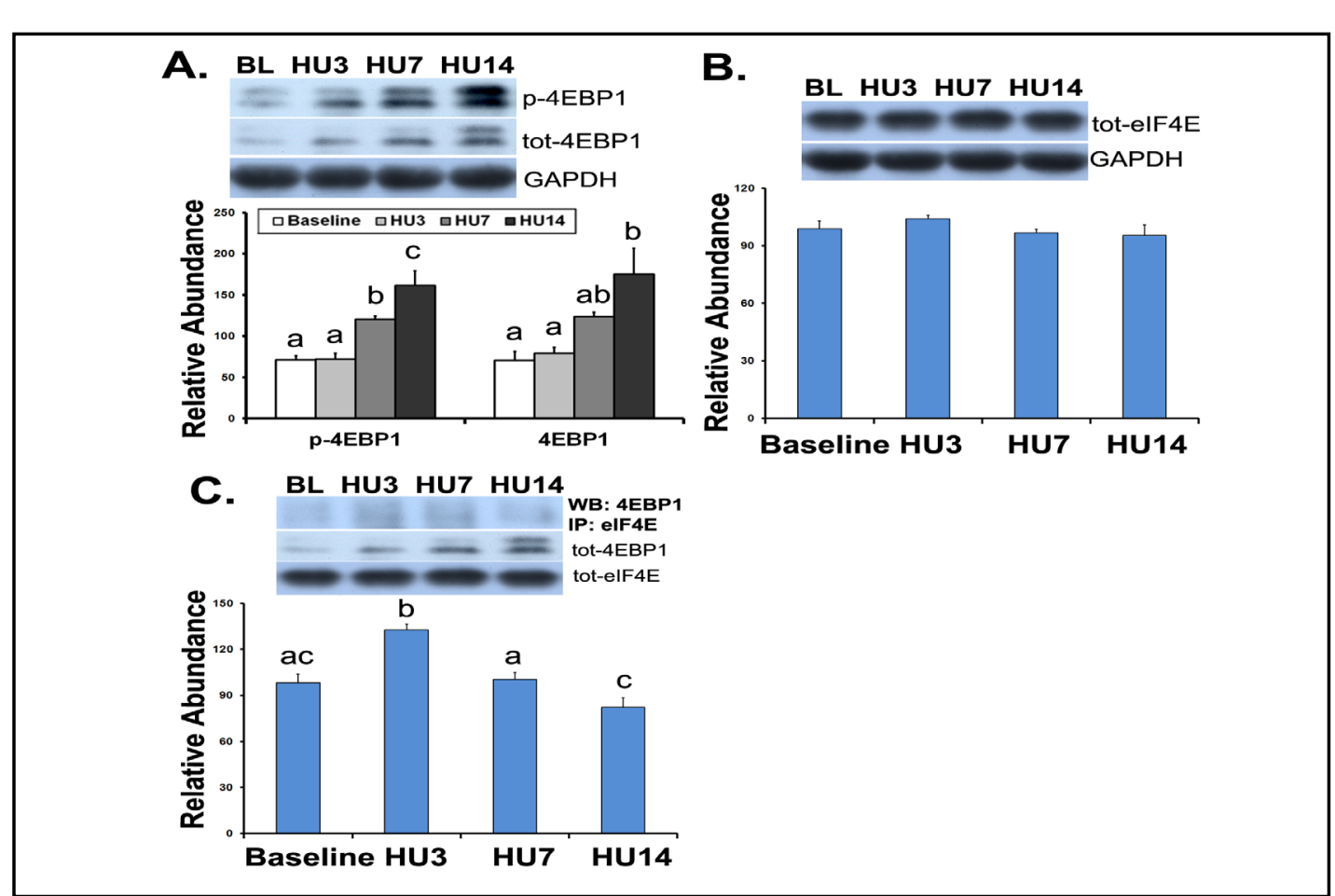

Fig. 6. Phosphorylation / inactivation of 4EBP1 and the binding of 4EBP1 to elF4E in gastrocnemius cytosolic proteins. A. Phosphorylation of 4EBP1 at Thr37/46 as determined by immunoblotting. B. Total eIF4E protein was determined by immunoblotting $(\mathrm{P}=0.3)$. C. Binding of 4EBP1 to elF4E was detected by co-immunoprecipitation. The eIF4E was immuno-precipitated and then 4EBP1 that bound to eIF4E was detected with immunoblotting. abc: Groups without the same letter are significantly different $(\mathrm{P} \leq 0.05)$.

in the 14-day HU animals, when compared to that in baseline control ( $\mathrm{P} \leq 0.05$; Fig. 5A). The phosphorylation of p70S6K-target S6 ribosomal protein at Ser235/236 was not changed in the 3- and 7-day HU muscles, while it was increased in the 14-day HU muscle ( $\mathrm{P} \leq 0.05$; Fig. 5B). Total proteins of both p70S6K and S6 ribosomal protein were decreased after 14 days of unloading ( $\mathrm{P} \leq 0.05$; Fig. 5).

\section{Phosphorylation of $4 E B P 1$ and association of $4 E B P 1$ with eIF4E}

Compared to that seen in control rats, gastrocnemius cytosolic total 4EBP1 protein was increased in the 14 days of $\mathrm{HU}$ rats $(\mathrm{P} \leq 0.05$; Fig. $6 \mathrm{~A})$. The abundance of phosphorylated 
4EBP1 (Thr37/46) in the day 7 and day $14 \mathrm{HU}$ rats was higher than that found in the control animals ( $\mathrm{P} \leq 0.05$; Fig. 6A). Co-immunoprecipitation experiments demonstrated that 4EBP1 bound to translational initiation factor eIF4E was increased in the gastrocnemius muscle of day $3 \mathrm{HU}$ rats when compared to baseline control animals of rats, while it was decreased in the 14-day HU muscle ( $\mathrm{P} \leq 0.05$; Fig. $6 \mathrm{C})$. The abundance of eIF4E total protein was not different between groups ( $\mathrm{P}=0.3$; Fig. $6 \mathrm{~B})$.

\section{Discussion}

Muscle disuse from extended bed rest or prolonged spaceflight / weightlessness is associated with muscle atrophy and impaired muscle function [1-3]. The adaptations of skeletal muscle to decreased mechanical loading are complex and involve changes in muscle mass, muscle protein expression and the transition from oxidative to glycolytic muscle fiber types $[1-3,19,20]$. It is thought that these changes in muscle phenotype are mediated by altering the relative balance between protein synthesis versus degradation [21-23]. Although short term muscle unloading has been shown to not alter the activation of Akt signaling in humans [24], how longer durations of muscle unloading may affect protein translational signaling such as the interaction between translational initiation factor eIF4E and 4EBP1 has not been investigated. Here we demonstrate that hindlimb unloading for 3 days significantly reduced cytosolic myosin content and increased the inhibitory binding of 4EBP1 to eIF4E, but not Akt/mTOR/p70S6K/S6 pathway signaling (Fig. 1, 3, 4, 5 and 6). Conversely, prolonged muscle disuse for 14 days was associated with the decreased myofiber size (CSA), and increases in the amount of soluble myosin which appeared, at least partially, to be mediated by the activation of Akt/mTOR/p70S6K/S6 signaling and the decreased repression of 4EBP1 on eIF4E. Taken together, these data suggest that the duration of muscle unloading has differential effects on the activation of protein translational signaling.

Consistent with earlier studies [1,2], we observed progressive decreases in body weight, muscle-to-body weight ratio and myofiber cross sectional area in the rat gastrocnemius muscle with increased duration of unloading (Fig. 1). In addition to changes in muscle mass and myofiber size, we also investigated the effects of muscle unloading on myosin expression given its pivotal role in modulating muscle contractility [25]. On the basis of prior work showing that sarcomeric remodeling occurs via the continually ordered diffusion of new monomeric myosin subunits into the existing sarcomere [26] we chose to examine how muscle unloading may regulate the total and cytoplasmic pools of myosin. Similar to previous report examining the regulation of myosin with hindlinb unloading in the gastrocnemius [27], our analysis of myosin expression using total protein extracts failed to show significant differences in whole myosin content with hindlimb unloading (Fig. 2). Conversely, when we examined the effects of hindlimb unloading on the amount of cytosolic myosin we found a significant decrease in acute phase (3 days of unloading) while we observed an increase of the amount of cytosolic myosin after seven and fourteen days of hindlimb unloading (Fig. 3). These results, when considered together with our findings using the total protein extracts, suggest that the acute response to muscle unloading is characterized by a rapid loss of muscle soluble contractile proteins while prolonged unloading is associated with increased myosin expression. The reasons for this response are not entirely clear. However, it is possible that this mechanism may have arisen to provide for the maintenance of contractile function in the presence of declining muscle mass and mechanical loading.

To examine whether other genes associated with the contractile apparatus might also be regulated in a similar fashion, we next performed gene expression profiling using microarrays containing $\sim 34,000$ genes. Consistent with others' work in unloaded soleus [19], our microarray analysis indicated that the abundance of several mRNA transcripts including muscle contractile proteins (such as myosin and actin) and regulatory proteins (such as nexilin and troponin) were increased over 2-fold in gastrocnemius muscle that had been subjected to 14 days of unloading when compared to that in HU3 muscle (Table 1). 
Liu/Blough/Arvapalli et al.: Muscle Disuse Changes Contractile Proteins and Translational Signaling

Fig. 7. Summary of alterations in muscle contractile proteins and protein translational signaling in response to acute and prolonged muscle disuse. When skeletal muscle experiences disuse, it initially increases the repression of 4EBP1 on translation initiation factor eIF4E, although the phosphorylation of Akt/mTOR/p70S6K/S6 pathway is unaltered (Acute phase of disuse; Brown square dot line). However, when muscle undergoes prolonged disuse and loses significant amount of muscle mass (atrophy), phosphorylation of both Akt/mTOR/ p70S6K/S6 and Akt/mTOR/4EBP1 pathways are activated, and the repression of 4EBP1 on eIF4E is decreased, resulting in increased expression of muscle contractile proteins to promote sarcomeric remodeling in atrophic muscle (Blue solid line). Open arrow $(\rightarrow)$ indicates increase or activation, while stopped line $\left(^{\perp}\right)$ suggests decrease or inhibition.

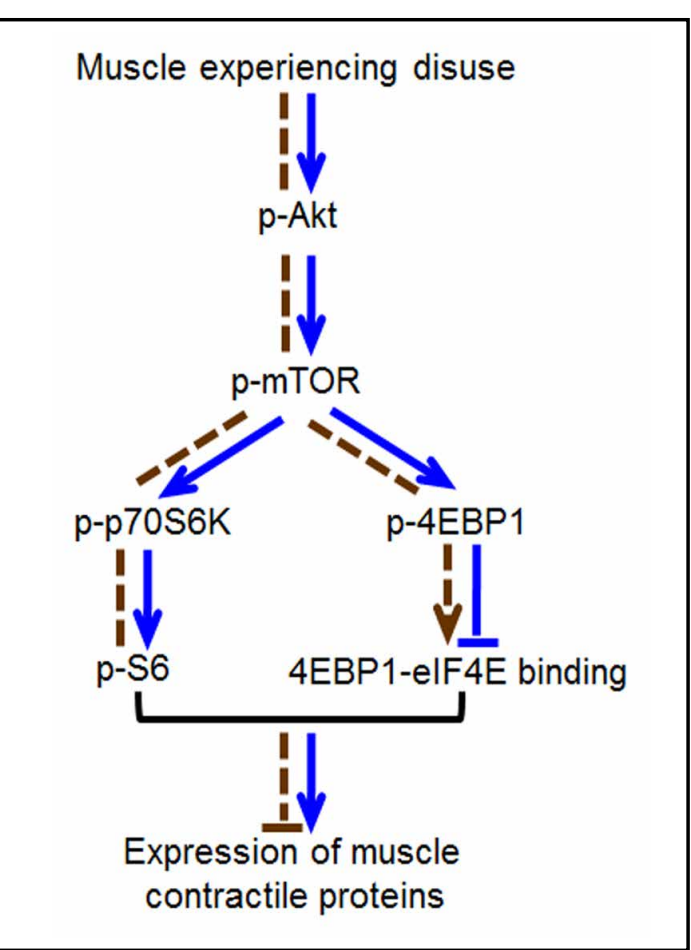

It is likely that several mechanisms may be involved in the regulation of muscle contractile protein levels during atrophic remodeling, including changes in protein degradation and synthesis [21-23]. Although a number of studies have examined the regulation of muscle protein degradation during muscle unloading, we chose to investigate the regulation of protein translational signaling given its pivotal role in muscle remodeling and re-growth. It has been suggested that nutritional status can affect protein synthesis $[28,29]$. To evaluate whether altered food consumption may have contributed to changes in muscle cytosolic contractile protein contents observed here, we measured food consumption during the unloading and calculated the amount of daily food intake. We found that 3-day unloaded animals had less daily food consumption than those 7- and 14-day unloaded rats (Fig. 1D), which is in parallel to those changes in muscle cytosolic contractile protein contents (Fig. 3 ). Given that mTOR signaling plays critical roles in nutrient sensing [28, 29], regulating protein synthesis and stimulating myocyte growth [4, 5], we focused on understanding how signaling through this pathway may be altered in response to muscle disuse. Akt kinase resides upstream of mTOR by phosphorylating the Ser 2448 residue of mTOR [30], while Akt enzyme activity is regulated by the phosphorylation of its Ser473 and Thr308 residues [13, 31]. Consistent with our observed changes in cytosolic myosin protein (Fig. 3B), we found that the phosphorylation of both Akt and mTOR appeared to be diminished during the acute phase of muscle disuse (0-3 days) before becoming increased during a prolonged disuse phase (7-14 days) (Fig. 4). Taken together, these data sugges that prolonged unloading eventually results in the reactivation of the Akt/mTOR pathway, where it might function to stimulate protein synthesis as a countermeasure to prevent further muscle loss.

To further understand the physiological significance of the increased Akt/mTOR activation seen in the 14-day unloaded muscle, we next determined the phosphorylation of two critical mTOR-substrates, p70S6K and 4EBP1. It is thought that the phosphorylation of p70S6K at Thr389 by mTOR functions to activate S6 ribosomal protein, a 40S ribosomal subunit component critical for the increased translation of ribosomal proteins and elongation factors required to promote the synthetic potential of the cell [6-8]. Supporting the changes that we observed in Akt/mTOR signaling, phosphorylation of both p70S6K on Thr389 and S6 
ribosomal protein on Ser235/236 was increased in the gastrocnemius muscles from 14-day unloaded rats (Fig. 5). Although the mechanism of how changes in ribosomal proteins and elongation factors modulate the activation of Akt / mTOR / p70S6K / S6 signaling are not well understood, our data suggest the increased activation of p70S6K / S6 signaling appears to compensate for the progressive loss of total 56 ribosomal protein with prolonged muscle unloading (Fig. 5B). Nonetheless, how this increase in Akt / mTOR / p70S6K / S6 signaling contributes to protein synthesis in disused muscle remains to be further determined.

Binding of the translation initiation factor eIF4E to the mRNA 5'-cap structure is critical for the initiation of protein translation $[32,33]$ and the process is inhibited when eIF4E is sequestered by its binding protein 4EBP1 [11]. Recent data have suggested that this repression can be relieved by the phosphorylation of 4EBP1 by mTOR which leads to the dissociation of 4EBP1 and eIF4E and activation of cap-dependent translation $[9,10]$. Consistent with our findings of increased phosphorylation of the Akt / mTOR / p70S6K / S6 pathway, we found that the phosphorylation of 4EBP1 at Thr37/46 was increased in the 7- and 14-day unloaded muscles (Fig. 6A). To examine the potential functional consequences of these data we next performed co-immunoprecipitation experiments to examine the association between eIF4E and 4EBP1. As expected from our 4EBP1 phosphorylation data, we found that the binding of 4EBP1 to eIF4E was increased in the 3-day unloaded muscle, while it was significantly decreased in the gastrocnemius muscles for the 14-day unloaded animals (Fig. 6C). This shift in 4EBP1-eIF4E interaction during muscle disuse to our knowledge has not been previously reported, but is consistent with other studies which examined the long term muscle atrophy seen with aging [16], chronic spinal cord-injury [34] and cancer cachexia [35], and supports the possibility that phosphorylation of the Akt/mTOR/4EBP1 pathway and the disassociation of the 4EBP1-eIF4E complex during prolonged unloading functions to stimulate protein synthesis and prevent further muscle loss (Fig. 7).

\section{Conclusions}

In summary, unloading-induced muscle atrophy is characterized by a biphasic protein translational signaling response. Specifically, the initial unloading phase (0-3 days) is associated with diminished cytosolic myosin content and repression of 4EBP1 on eIF4E, while prolonged disuse atrophy is associated with increased expression of myosin and other myofibrillar proteins and the activation of translational signaling, including activation of Akt/mTOR/p70S6K/S6 pathway and diminished inhibition of eIF4E by 4EBP1 sequestration (Fig. 7). These findings provide new insight into how protein translational signaling is modulated during atrophic remodeling and may lead to new countermeasures to diminish the deleterious effects produced by prolonged muscle disuse.

\section{Acknowledgements}

This work was supported by grants provided by the Department of Energy grant \#DESC0005162 and the NASA-EPSCoR to ERB, and the NASA NNX07AL53A to MW. The authors would like to acknowledge the support of the Huntington VA Medical Center for laboratory space and equipment. The monoclonal antibody MF20 (contributed by Dr. Donald A Fischman) was obtained from the Developmental Studies Hybridoma Bank, developed under the auspices of the Eunice Kennedy Shriver National Institute of Child Health \& Development and maintained by the Department of Biology at The University of Iowa. 
Liu/Blough/Arvapalli et al.: Muscle Disuse Changes Contractile Proteins and Translational Signaling

\section{References}

1 Fitts RH, Metzger JM, Riley DA, Unsworth BR: Models of disuse: A comparison of hindlimb suspension and immobilization. J Appl Physiol 1986;60:1946-1953.

2 Riley DA, Slocum GR, Bain JL, Sedlak FR, Sowa TE, Mellender JW: Rat hindlimb unloading: Soleus histochemistry, ultrastructure, and electromyography. J Appl Physiol 1990;69:58-66.

3 Morey-Holton ER, Globus RK: Hindlimb unloading rodent model: Technical aspects. J Appl Physiol 2002;92:1367-1377.

4 Bodine SC, Stitt TN, Gonzalez M, Kline WO, Stover GL, Bauerlein R, Zlotchenko E, Scrimgeour A, Lawrence JC, Glass DJ, Yancopoulos GD: Akt/mtor pathway is a crucial regulator of skeletal muscle hypertrophy and can prevent muscle atrophy in vivo. Nat Cell Biol 2001;3:1014-1019.

5 Wu M, Falasca M, Blough ER: Akt/protein kinase b in skeletal muscle physiology and pathology. J Cell Physiol 2011;226:29-36.

6 Jefferies HB, Reinhard C, Kozma SC, Thomas G: Rapamycin selectively represses translation of the “Polypyrimidine tract” Mrna family. Proc Natl Acad Sci USA 1994;91:4441-4445.

$7 \quad$ Ferrari S, Thomas G: S6 phosphorylation and the p70s6k/p85s6k. Crit Rev Biochem Mol Biol 1994;29:385413.

8 Weng QP, Kozlowski M, Belham C, Zhang A, Comb MJ, Avruch J: Regulation of the p70 s6 kinase by phosphorylation in vivo. Analysis using site-specific anti-phosphopeptide antibodies. J Biol Chem 1998;273:16621-16629.

9 Gingras AC, Gygi SP, Raught B, Polakiewicz RD, Abraham RT, Hoekstra MF, Aebersold R, Sonenberg N: Regulation of 4e-bp1 phosphorylation: A novel two-step mechanism. Genes Dev 1999;13:1422-1437.

10 Brunn GJ, Hudson CC, Sekulic A, Williams JM, Hosoi H, Houghton PJ, Lawrence JC Jr, Abraham RT: Phosphorylation of the translational repressor phas-i by the mammalian target of rapamycin. Science 1997;277:99-101.

11 Pause A, Belsham GJ, Gingras AC, Donze O, Lin TA, Lawrence JC Jr, Sonenberg N: Insulin-dependent stimulation of protein synthesis by phosphorylation of a regulator of 5 '-cap function. Nature 1994;371:762-767.

12 Wang Y, Wu M, Al-Rousan R, Liu H, Fannin J, Paturi S, Arvapalli R, Katta A, Kakarla S, Rice K, Triest WE, Blough ER: Iron-induced cardiac damage: Role of apoptosis and deferasirox intervention. J Pharmacol Exp Ther 2011;336:1-8.

13 Wu M, Katta A, Gadde MK, Liu H, Kakarla SK, Fannin J, Paturi S, Arvapalli RK, Rice KM, Wang Y, Blough ER: Aging-associated dysfunction of akt/protein kinase b: S-nitrosylation and acetaminophen intervention. PLoS One 2009;4:e6430.

14 Blough ER, Rennie ER, Zhang F, Reiser PJ: Enhanced electrophoretic separation and resolution of myosin heavy chains in mammalian and avian skeletal muscles. Anal Biochem 1996;233:31-35.

15 Bicer S, Reiser PJ: Myosin light chain isoform expression among single mammalian skeletal muscle fibers: Species variations. J Muscle Res Cell Motil 2004;25:623-633.

16 Wu M, Liu H, Fannin J, Katta A, Wang Y, Arvapalli RK, Paturi S, Karkala SK, Rice KM, Blough ER: Acetaminophen improves protein translational signaling in aged skeletal muscle. Rejuvenation Res 2010;13:571-579.

17 Wu M, Desai DH, Kakarla SK, Katta A, Paturi S, Gutta AK, Rice KM, Walker EM Jr, Blough ER: Acetaminophen prevents aging-associated hyperglycemia in aged rats: Effect of aging-associated hyperactivation of p38mapk and erk1/2. Diabetes Metab Res Rev 2009;25:279-286.

18 Covi JA, Bader BD, Chang ES, Mykles DL: Molt cycle regulation of protein synthesis in skeletal muscle of the blackback land crab, gecarcinus lateralis, and the differential expression of a myostatin-like factor during atrophy induced by molting or unweighting. J Exp Biol 2010;213:172-183.

19 Stevens L, Bastide B, Kischel P, Pette D, Mounier Y: Time-dependent changes in expression of troponin subunit isoforms in unloaded rat soleus muscle. Am J Physiol Cell Physiol 2002;282:C1025-1030.

20 Pandorf CE, Haddad F, Wright C, Bodell PW, Baldwin KM: Differential epigenetic modifications of histones at the myosin heavy chain genes in fast and slow skeletal muscle fibers and in response to muscle unloading. Am J Physiol Cell Physiol 2009;297:C6-16.

21 Hornberger TA, Hunter RB, Kandarian SC, Esser KA: Regulation of translation factors during hindlimb unloading and denervation of skeletal muscle in rats. Am J Physiol Cell Physiol 2001;281:C179-187. 


\section{Cellular Physiology Cell Physiol Biochem 2012;30:1202-1214 and Biochemistry \\ Liu/Blough/Arvapalli et al.: Muscle Disuse Changes Contractile Proteins and Translational Signaling}

22 Childs TE, Spangenburg EE, Vyas DR, Booth FW: Temporal alterations in protein signaling cascades during recovery from muscle atrophy. Am J Physiol Cell Physiol 2003;285:C391-398.

23 Franch HA, Price SR: Molecular signaling pathways regulating muscle proteolysis during atrophy. Curr Opin Clin Nutr Metab Care 2005;8:271-275.

24 Gustafsson T, Osterlund T, Flanagan JN, von Walden F, Trappe TA, Linnehan RM, Tesch PA: Effects of 3 days unloading on molecular regulators of muscle size in humans. J Appl Physiol 2010;109:721-727.

25 Cheney RE, Riley MA, Mooseker MS: Phylogenetic analysis of the myosin superfamily. Cell Motil Cytoskeleton 1993;24:215-223.

26 Russell B, Wenderoth MP, Goldspink PH: Remodeling of myofibrils: Subcellular distribution of myosin heavy chain mrna and protein. Am J Physiol 1992;262:R339-345.

27 Schuenke MD, Reed DW, Kraemer WJ, Staron RS, Volek JS, Hymer WC, Gordon S, Perry Koziris L: Effects of 14 days of microgravity on fast hindlimb and diaphragm muscles of the rat. Eur J Appl Physiol 2009;106:885-892.

28 Gleason CE, Lu D, Witters LA, Newgard CB, Birnbaum MJ: The role of ampk and mtor in nutrient sensing in pancreatic beta-cells. J Biol Chem 2007;282:10341-10351.

29 Fujita S, Dreyer HC, Drummond MJ, Glynn EL, Cadenas JG, Yoshizawa F, Volpi E, Rasmussen BB: Nutrient signalling in the regulation of human muscle protein synthesis. J Physiol 2007;582:813-823.

30 Nave BT, Ouwens M, Withers DJ, Alessi DR, Shepherd PR: Mammalian target of rapamycin is a direct target for protein kinase $b$ : Identification of a convergence point for opposing effects of insulin and amino-acid deficiency on protein translation. Biochem J 1999;344 Pt 2:427-431.

31 Scheid MP, Marignani PA, Woodgett JR: Multiple phosphoinositide 3-kinase-dependent steps in activation of protein kinase b. Mol Cell Biol 2002;22:6247-6260.

32 Sonenberg N, Morgan MA, Merrick WC, Shatkin AJ: A polypeptide in eukaryotic initiation factors that crosslinks specifically to the 5'-terminal cap in mrna. Proc Natl Acad Sci USA 1978;75:4843-4847.

33 Gingras AC, Raught B, Sonenberg N: Eif4 initiation factors: Effectors of mrna recruitment to ribosomes and regulators of translation. Annu Rev Biochem 1999;68:913-963.

34 Leger B, Senese R, Al-Khodairy AW, Deriaz O, Gobelet C, Giacobino JP, Russell AP: Atrogin-1, murf1, and foxo, as well as phosphorylated gsk-3beta and 4e-bp1 are reduced in skeletal muscle of chronic spinal cord-injured patients. Muscle Nerve 2009;40:69-78.

35 Eley HL, Russell ST, Tisdale MJ: Effect of branched-chain amino acids on muscle atrophy in cancer cachexia. Biochem J 2007;407:113-120. 